\title{
G. Schmid-Ott
}

S. Schallmayer

\section{Psychosoziale Konsequenzen der Psoriasis}

\section{Psychosocial Consequences of Psoriasis}

\section{Zusammenfassung}

Zwei wesentliche psychosoziale Felder sind bei der Psoriasis von Relevanz: zum einen psychoneuroimmunologische Aspekte und zum anderen somatopsychische Gesichtspunkte, d.h. vor allem die aus der Erkrankung oft resultierende gravierende Minderung der Lebensqualität bzw. die damit verbundene Krankheitslast. Psychosoziale Konsequenzen der Psoriasis treten teilweise auch bei eher kleiner Fläche der erkrankten Hautstellen auf. Auch empirische Befunde sprechen dafür, dass die behandelnden Dermatologen die psychosozialen Belastungen durch problematische Interaktionen in öffentlichen Situationen und im Sexualleben auch bei relativ geringer Ausdehnung der Hauterkrankung im Patienten-Kontakt thematisieren. Denn selbst die Erkrankung der „unsichtbaren Regionen“ hat häufig erhebliche psychosoziale Einschränkungen, Stigmatisierungsgefühle und soziale Ausgrenzungen zur Folge. Diese Konsequenzen können durch die Teilnahme an Patientenschulungen, die durch ein interdisziplinäres Team der Fachgebiete Dermatologie, Psychologie bzw. Psychosomatische Medizin und Psychotherapie sowie Ökotrophologie durchgeführt werden bzw. an einer Selbsthilfegruppe gemindert werden.
Abstract

Two psychosocial fields are of special relevance in psoriasis: first, psychoneuroimmunological aspects and, second, somatopsychic aspects, i.e. the negative impact on quality of life and the burden caused by this skin disease.

Psychosocial consequences of psoriasis might already emerge with only small patches of skin being affected. Empirical data indicate that treating dermatologists should address possible negative effects elicited by problematic encounters with the public and in sexual relationships even if the severity of the disease is low as lesions on invisible parts of the body can already cause serious adverse impairment, the experience of stigmatisation, and social exclusion of patients. Such psychosocial consequences can be reduced by partaking in a patient-education programme specially designed by a multidisciplinary team of dermatologists, psychologists, specialists in psychosomatic medicine, psychotherapy as well as dieticians and by attending a self-help group respectively.
In der Diskussion über psychosomatische bzw. somatopsychische Gesichtspunkte der Schuppenflechte wurden in den letzten Jahren vor allem letztere Aspekte, d.h. die psychosozialen Konsequenzen im Kontext dieser chronischen bzw. chronisch- rezidivierenden Erkrankung untersucht; allerdings haben auch experimentelle Studien mit einem Laborstressor zunehmend an Bedeutung gewonnen. 
Psychosomatische und psychoneuroimmunologische Aspekte der Psoriasis

Psychosomatische Hypothesen einer „spezifischen Persönlichkeit" bzw. einer vermehrten prämorbiden Komorbidität von psychischen Störungen, einer generell vermehrten Ängstlichkeit bzw. Depressivität, eines veränderten Umgangs mit Aggressivität, einer verminderten sozialen Anpassung oder einer speziellen Konstellation von Abwehrmechanismen bei Patientinnen und Patienten mit Psoriasis fanden in bisherigen Studien keine Bestätigung [1]. Ebenso erbrachten verschiedene Studien zum Einfluss von Life Events auf Erstmanifestation oder Rezidive der Psoriasis wenig konsistente Ergebnisse [2].

Bei der Psoriasis handelt es sich um keine „psychosomatische Erkrankung“, sondern vielmehr um eine multifaktoriell bedingte Erkrankung, bei deren Auslösung bzw. Verlauf sehr unterschiedliche Umwelteinflüsse, u.U. auch psychischer Stress, von Relevanz sein können. Allerdings existiert bis jetzt erst eine begrenzte Anzahl experimenteller Studien, welche mit einem standardisierten Ansatz den Einfluss von psychologischem Stress auf für die Schuppenflechte relevante psychobiologischen Links bei Psoriasis-Patientinnen und -Patienten im Vergleich zu gesunden Kontrollpersonen bestimmen [3-6]. Reduzierte stressinduzierte Cortisolkonzentrationen bei Psoriasis-Patienten im Vergleich zu gesunden Kontrollpersonen sprechen für die Modulation der Hypothalamus-Hypophysen-Nebennierenrinden-Achse auch bei dieser Erkrankung, bei der eine zugunsten der Th1-Zellen verschobene Th1/Th2-Balance zu finden ist [3]. Der Befund eines signifikanten stressinduzierten Anstiegs von zytolytischen $C D 8^{+}$ / CD16- / CD11 b+ T-Lymphozyten während eines kurz andauernden psychischen Stresses und ihr Abfall eine Stunde danach im Blut von Patienten mit Psoriasis, nicht jedoch bei gesunden Kontrollpersonen, sprechen für den Einfluss eines psychologischen Stressors auf für die Schuppenflechte pathophysiologisch relevante Parameter [4].

\section{Somatopsychische Aspekte der Psoriasis}

Im Kontext somatopsychischer Gesichtspunkte wird insbesondere die durch die Psoriasis häufig entstehende gravierende Minderung der Lebensqualität zunehmend diskutiert [7]. Die gestiegene Bedeutung des Konzeptes der Lebensqualität in PsoriasisStudien zeigt, dass der somatopsychische Ansatz den bedeutsamsten Aspekt der Psychodermatologie darstellt [8]. Von Ergebnissen ihrer Studie, an der 6194 Psoriasis-Patienten teilnahmen, berichten Krueger et al. [9], dass 79\% angaben, in ihrem Alltag negative Konsequenzen durch die Erkrankung zu erfahren. Durch Chronizität und Unvorhersehbarkeit des Verlaufes der Psoriasis fühlen sich Patienten der Krankheit häufig ausgeliefert. Das Aufbringen von Geduld und Zeit bei der Behandlung stellt eine weitere Herausforderung dar. Nicht zuletzt erleben Betroffene subjektiv eine Beeinträchtigung ihrer Attraktivität, wobei der Befall sichtbarer Körperstellen die Beziehung zur Umwelt erschwert, in der sie häufig Ablehnung, Schuldzuweisungen und Vorurteile erleben. Oftmals leiden Patienten mehr unter den psychosozialen Konsequenzen als unter den körperlichen Krankheitssymptomen selbst [10]. Dies wird besonders im Nachstehenden deutlich:
„Wegen meiner Haut schloss ich alle Berufe für mich aus, die ein präsentables Äußeres verlangen - Geschäftsmann, Lehrer, Bankfachmann, Filmstar. (...) Warum habe ich so jung geheiratet? Weil ich, kaum dass ich eine hübsche Frau gefunden hatte, die mir meine Haut verzieh, nicht riskieren wollte, sie zu verlieren und mir eine andere suchen zu müssen “ [11].

Große Stichproben erlauben die differenzierte empirische Betrachtung der Zusammenhänge zwischen Psoriasis und (nach Angaben der Untersuchten jemals diagnostizierten) Zweiterkrankungen, z.B. mittels des „Psoriasis Disability Index“ (PDI [12]). Der PDI beinhaltet 15 Aussagen zur Messung der mit der Psoriasis assoziierten psychosozialen, aber auch körperlichen Einschränkungen in den letzten vier Wochen vor der Untersuchung mit vier Abstufungen: „gar nicht“, ,selten“, ,oft“ und „sehr oft" (Rangfolge 0 bis 45). Bei ca. 50\% der 3753 Teilnehmer der Studie lag eine „leichte“ (PDI = 9), bei ca. $35 \%$ eine „mittelschwere" (PDI $=14)$ und bei ca. $15 \%$ eine "schwere“ Psoriasis $($ PDI = 19) vor ([13], vgl. Tab.1). In dieser Studie wurden auch die Schwierigkeiten der Betroffenen vor allem bei der Beeinflussung der Kleidungswahl, beim Frisör, im Schwimmbad, beim Sport und im Sexualleben auch schon bei relativ geringer Ausdehnung der Hauterkrankung deutlich [13]. Außerdem legt eine frühere Studie nahe, dass selbst das Betroffensein auch „unsichtbarer Regionen“ (Achselhöhlen, Arme, Rücken, Ellbogen, Knie, Füße, Beine und Oberkörper) eine erhebliche psychosoziale Bedeutung für die Psoriasis-Patientinnen bzw. -Patienten hat [14].

Tab. 2 stellt den Einfluss verschiedener Komorbiditäten in der beschriebenen Stichprobe dar. Nur ein Lupus erythematodes ( $0.3 \%$ der Erkrankten) hatte keinen signifikanten Einfluss auf den PDI-Wert bzw. der PDI war beim Vorliegen eines Diabetes mellitus lediglich tendenziell höher als ohne diese Erkrankung. Beim Vorliegen der übrigen, im Folgenden genannten Zweiterkrankungen ergaben sich signifikant ausgeprägtere psychosoziale und körperliche Einschränkungen bzw. Behinderungen im PDI: als ohne eine entsprechende Komorbidität: Arthritis (ca. $38 \%$ ), allgemeiner Gelenkschmerz (ca. 50\%), Morbus Crohn (ca. $4 \%$ ), Herzerkrankung (ca. 11\%), Adipositas (ca. 10\%), Zoster (ca. $7 \%$ ) und Autoimmunerkrankung (ca. $5 \%$ ); besonders deutlich waren die Unterschiede der PDI-Mittelwerte bei einer Depression (ca. 14\%) mit einer durchschnittlichen Erhöhung des PDIWertes um 57,8\% (17.2 versus 10.9) und einer Fibromyalgie (ca. $2 \%$ ) mit einer Erhöhung um 61,5\% (18.9 versus 11.7). Diese Ergebnisse sind nur eingeschränkt interpretierbar, da es sich um Patientenselbstangaben handelt und z. B. nicht zwischen Lupus erythematodes und Autoimmunerkrankungen bzw. zwischen Arthritis und Gelenkschmerz eindeutig differenziert wird. Trotzdem sind die Ergebnisse unseres Erachtens aufgrund der großen Fallzahl und der Art der Stichprobe trotz dieser Einschränkung von Bedeutung.

Die Krankheitslast der Psoriasis sollte also besonders bei den Zweiterkrankungen Depression und Fibromyalgie beachtet werden. In diesem Zusammenhang ist festzuhalten, dass auch die Komorbidität zwischen der Fibromyalgie und depressiven Störungen hoch ist: die Lebenszeitprävalenz für eine zusätzliche schwere depressive Episode oder eine schwere Episode einer rezidivierenden depressiven Störung bei der Fibromyalgie beträgt $68 \%[15]$. 
Tab. 1 EUROPSO Psoriasis Patient Survey - Deutschland ( $n$ = 3753. Rücklaufquote ca. 42,3\% (nach [13])

\begin{tabular}{|c|c|c|c|}
\hline Geschlecht & F: $43,9 \%$ & M: 55,9\% & k. A.: $0,2 \%$ \\
\hline Alter (Jahre) & $54,3( \pm 13,0)$ & & \\
\hline Art der Erkrankung & Psoriasis vulgaris & & $93,5 \%$ \\
\hline Dauer der Erkrankung (Jahre) & $31,4( \pm 14,4)$ & & \\
\hline $\begin{array}{l}\text { Ausmaß der erkrankten } \\
\text { Körperoberfläche } \times \% *\end{array}$ & $\begin{array}{l}\text { keine/leichte Psoriasis } \\
\text { (<3\% Körperoberfläche) } \\
49,2 \%\end{array}$ & $\begin{array}{l}\text { Mittelschwere Psoriasis } \\
\text { (3-10\% Körperoberfläche) } \\
36,5 \%\end{array}$ & $\begin{array}{l}\text { Schwere Psoriasis } \\
\text { (>10\% Körperoberfläche) } \\
14,4 \%\end{array}$ \\
\hline $\begin{array}{l}\text { Psoriasis Disability Index (PDI) } \\
\text { Mittelwert = } 12 \\
\text { (nach Finlay et al., 1990, Range } 0-45 \text { ) }\end{array}$ & $9^{* *}$ & $14^{* *}$ & $19^{* *}$ \\
\hline
\end{tabular}

* auswertbar: $\mathrm{n}=3673 ;{ }^{* *}$ ANOVA: $\mathrm{p}<0.001$

Tab. 2 Krankheitslast durch Psoriasis und Zweiterkrankungen

\begin{tabular}{|c|c|c|c|c|c|c|}
\hline & \multicolumn{3}{|c|}{ PDI* mit Zweiterkrankung } & \multicolumn{2}{|c|}{ PDI ohne Zweiterkrankung } & \multirow[b]{2}{*}{$P$ (t-Test) } \\
\hline & $\%$ & Mittel & $S D$ & Mittel & $S D$ & \\
\hline Arthritis & 37,5 & 14 & 9 & 10,7 & 7,9 & $<0,001$ \\
\hline Depression & 14,4 & 17,2 & 9,6 & 10,9 & 8,4 & $<0,001$ \\
\hline Morbus Crohn & 3,5 & 14,8 & 9,9 & 11,7 & 8,4 & $<0,001$ \\
\hline Diabetes mellitus & 6,6 & 12,8 & 8,4 & 11,8 & 8,5 & 0,06 \\
\hline Herzerkrankung & 11,1 & 13,6 & 8,8 & 11,6 & 8,4 & $<0,001$ \\
\hline Fettleibigkeit & 10,1 & 14,6 & 9,1 & 11,5 & 8,4 & $<0,001$ \\
\hline Gürtelrose & 6,7 & 13,2 & 8,3 & 11,7 & 8,5 & $<0,01$ \\
\hline Lupus erythematodes & 0,3 & 8,6 & 6,8 & 11,9 & 8,5 & 0,17 \\
\hline Autoimmunerkrankung & 5,1 & 15,3 & 10 & 11,7 & 8,4 & $<0,001$ \\
\hline
\end{tabular}

* Psoriasis Disability Index (nach [12])

Neben der Krankheitsbewältigung [16] und krankheitsbedingten Beeinträchtigungen der Lebensqualität stellt das Stigmatisierungsgefühl, d.h. die soziale Entwertung und Ausgrenzung im Zusammenhang mit einer sichtbaren oder in Beziehungen relevanten Hautsymptomatik [13], eines der wesentlichen Konzepte zur Erfassung der psychosozialen Krankheitsfolgen der Psoriasis dar. Erfahrungen aus der Praxis deuten jedenfalls darauf hin, dass die Erkrankung, unabhängig von möglichen Ursachen für Erstmanifestation und Exazerbation, deutliche psychosoziale Konsequenzen für die meisten der Betroffenen zur Folge hat, welche sich in den Bereichen Lebensqualität, Stigmatisierung, Alltagsstress, Behinderung und Krankheitsverarbeitung zeigen. Hierbei wird nicht nur von Dermatologen der häufig entstehenden Stigmatisierung eine besondere Bedeutung beigemessen [1]. Psychosoziale Konsequenzen für Betroffene entstehen dabei im Sozialkontakt, d.h. in der unvermeidbaren Interaktion mit der Umwelt, wie sich im Folgenden darstellt:

„Patienten mit Psoriasis, besonders jüngere Menschen, sind oft durch Psoriasis psychisch sehr stark belastet und in ihrem sozialen Verhalten erheblich gehemmt. Mitmenschen empfinden die Hauterscheinungen als unästhetisch oder glauben, sie seien ansteckend. Vielfach werden solche Patientin in Sportstätten, Schwimmbädern oder Kurbädern zurückgewiesen“ [17].
In dieser Aussage gehen die Autoren auf zwei zentrale, sich nur teilweise überschneidende Aspekte der Stigmatisierung ein: Erstens nehmen sich die Patienten selbst auf eine bestimmte Art und Weise wahr (,ich bin stigmatisiert“), wobei das Verhalten der Umwelt und ihr interaktioneller Druck bzw. die projektive Unterstellung einer Entwertung in unterschiedlich starker Ausprägung relevant sind. Zweitens stellen die Betroffenen den „Gegenstand“ relativ oder völlig unmissverständlicher Äußerungen und Handlungen der „Normalen“ („ich werde stigmatisiert“) dar. Stern et al. [18] zufolge geht die Stigmatisierung bereits bei geringer „objektiver“ Schwere mit einer erheblichen psychosozialen Krankheitslast einher.

Die Gruppe der Psoriasis-Patienten, bei der die Indikation für eine ergänzende psychosomatische Behandlung besteht, ist gegenüber einer Psychotherapie oft skeptisch eingestellt. Es spricht klinisch viel für die Annahme, dass hierdurch häufig eine Doppelstigmatisierung entsteht: zum einen durch die Dermatose (meist sichtbares Stigma) und zum anderen durch die häufig mit psychosozialen Problemen bzw. deren Behandlung einhergehende Diskriminierung (unsichtbares Stigma). Die klinische Bedeutung des Stigmatisierungsgefühls von Patienten, die an einer Psoriasis leiden, wird bereits seit den 80er Jahren in der psychodermatologischen Literatur empirisch untersucht, z.B. $[10,13,19-23]$. 
Die mit Psoriasis assoziierten psychosozialen und körperlichen Einschränkungen bzw. Behinderungen (z.B. messbar mit dem „Psoriasis Disability Index“, PDI [12]) tragen - wie bei vielen anderen chronischen somatischen Störungen - wesentlich zu der durch die Hauterkrankung entstehenden Belastung bei. Zudem erhöht sich speziell bei chronischen Dermatosen die durch die Hauterkrankung ausgelöste Belastung zusätzlich durch Stigmatisierung, z.B. [22,24,25], welche bei der Psoriasis hoch mit dem so genannten krankheitsspezifischen Alltagsstress („daily hassles“) korreliert [26]. Interessant ist in diesem Zusammenhang außerdem, dass Stigmatisierungsgefühl und Lebensqualität von Psoriasis- und Neurodermitis-Patienten mit vergleichbaren soziodemographischen und Krankheits-Charakteristika nicht signifikant unterschiedlich sind [10].

Besonders bemerkenswert ist die Tatsache, dass allein durch die Antizipation potenziell stigmatisierender Situationen diese häufig konsequent vermieden werden (z. B. in die Sauna gehen), was zwar dazu führt, dass sich Psoriasis-Patienten seltener diskriminiert fühlen, aber gleichzeitig eine Einschränkung ihrer sozialen Aktivitäten bedeutet [28]. Darüber hinaus spielt die Häufigkeit entsprechender Situationen eine geringere Rolle für die subjektiv erlebten psychosozialen Konsequenzen. So kann z.B. die einem Erkrankten in der Öffentlichkeit einmalig gestellte Frage „Ist es AIDS?“ lange andauernde Auswirkungen haben [13]. Daraus kann jedoch nicht die Schlussfolgerung gezogen werden, dass bei dieser Vermeidung eine besonders stark ausgeprägte soziale Angst oder gar eine soziale Phobie vorliegt. Vielmehr handelt es sich um eine in der Regel angemessene Befürchtung bezüglich einer potentiellen Reaktion Außenstehender, welche durch die eigene Einstellung oder das eigene Verhalten primär nicht beeinflussbar ist, es sei denn, die krankhaft veränderten Hautstellen werden so verdeckt, dass sie unsichtbar sind. Das Tragen einer Bekleidung, welche die Extremitäten bedeckt, fällt sehr stark auf, wenn alle anderen sich betont leicht kleiden, z.B. im Sommer und während der Freizeit. Auswirkungen der selbst gewählten Einschränkung, ohne dass unbedingt viele „dramatische“ Ereignisse (für Außenstehende oft „banale“ Interaktionen) stattgefunden haben müssen, können die Betroffenen stark belasten. Dies macht deutlich, dass die Psoriasis zweifelsohne häufig interaktionell relevant ist, was ebenso bei sexuellen Beziehungen eine große Rolle spielen kann.

\section{Somatopsychische Aspekte der Psoriasis im Vergleich zu} anderen chronischen Erkrankungen

Vor dem beschriebenen Hintergrund sind die auf den ersten Blick erstaunlichen Befunde der US-amerikanischen Studie von Rapp et al. [27] weniger überraschend: Danach war der Einfluss der Schuppenflechte auf die Lebensqualität der Betroffenen ebenso ausgeprägt wie der von anderen chronischen und zum Teil lebensbedrohlichen Krankheiten (z.B. Krebserkrankungen, Herzinfarkte und chronische Lungenerkrankungen). Nur Patienten mit einer dekompensierten Herzinsuffizienz hatten danach eine deutlich stärkere Beeinträchtigung der körperlichen Gesundheit. Eine wesentlich stärkere Einschränkung der seelischen Gesundheit wurde lediglich bei Patienten mit Depressionen gefunden. Dies ist am ehesten vor dem Hintergrund der erheblichen mit der Psoriasis verbundenen Krankheitslast zu erklären.
Psoriasisschulung

Aufgrund der komplexen psychosozialen Folgen chronischer Dermatosen ist die adäquate Therapie der Patienten von großer Bedeutung, wobei insbesondere die Bereiche Krankheitsmanagement und Patienten-Empowerment gefördert werden sollten. Hierbei spielen Patientenschulungen eine wichtige Rolle, um das Selbstbewusstsein zu stärken und um psychosozialen Konsequenzen der Psoriasis entgegen zu wirken.

In der modernen Psoriasistherapie spielt das dynamische Konzept des Selbstmanagements eine bedeutende Rolle: Ein angemessenes Krankheitskonzept bei Psoriasis beinhaltet sowohl adäquate Vorstellungen über Ursachen und Risiken der bzw. Belastungen durch die Hautkrankheit, als auch wesentliche Faktoren, die die aktuelle Lebensqualität des Patienten beeinflussen. Andererseits stellt es die Voraussetzung für ein angemessenes Behandlungskonzept dar, welches wiederum einerseits das Krankheitsmanagement über den subjektiv vermuteten Nutzen der medikamentösen Behandlung, selbst eingeschätzte eigene Handlungskompetenzen und vorliegende Ressourcen zur Krankheitsbewältigung beeinflusst, andererseits die Bereitschaft des Patienten zur langfristigen, eigenverantwortlichen Therapiemitarbeit fördert. Eine solche Kooperation fördert Selbstwirksamkeitserfahrungen des Patienten im Umgang mit der Krankheit [29] und resultiert in gesteigertem „Patienten-Empowerment“. Schließlich ist auch im Zusammenhang mit neuartigen somatischen Behandlungsansätzen der Psoriasis mit Biologicals [30] eine gute Kooperation von Dermatologen und Patienten unabdingbar, um die Zahl der Behandlungsabbrecher und damit das individuelle Leiden, die psychosozialen Konsequenzen, aber auch die Kosten der Erkrankung möglichst gering zu halten. Dabei wird der Psoriasis-Schulung eine besondere Bedeutung beigemessen. Für Betroffene mit verschiedenen Arten von chronischen Erkrankungen (z. B. Diabetes, Asthma bronchiale und Neurodermitis) gibt es solche Patientenschulungsprogramme bereits seit mehreren Jahren; die Kosten werden von den Krankenkassen getragen. Auch für an Schuppenflechte erkrankte Menschen wurde ein solches Schulungsprogramm entwickelt, das sich an erwachsene Psoriasis-Patienten oder Eltern von erkrankten Kindern richtet und neben der Aufklärung über die somatischen und psychosozialen Einflussfaktoren der Erkrankung auch sozialrechtliche Aspekte, Entspannungstechniken und Bewältigungsstrategien beinhaltet. Ziel dieser Schulung, die durch ein multiprofessionelles Team der Fachgebiete Dermatologie, Psychologie bzw. Psychosomatische Medizin und Psychotherapie sowie Ökotrophologie geleitet wird, ist es, Krankheitsschübe zu vermindern und praktische Umgangsmöglichkeiten mit den Krankheitssymptomen zu vermitteln.

\section{Selbsthilfeorganisationen}

Darüber hinaus stellt der Austausch der Betroffenen untereinander im Sinne der Selbsthilfe einen ebenso wichtigen Punkt in der optimalen Versorgung von Psoriasis-Patienten dar. Besonders für Patienten, die bisher keine Kontakte zu Selbsthilfegruppen aufgenommen haben, ist es in der Regel eine gute Erfahrung, mit ebenfalls Betroffenen offener und in anderer Art und Weise sprechen zu können, als dies mit Behandlern, nicht betroffenen An- 
gehörigen und Freunden der Fall ist. Daher wird Selbsthilfe von den meisten als entlastend und als Bereicherung erlebt und kann direkt dazu beitragen, psychosoziale Konsequenzen der Erkrankung durch eine bessere Krankheitsverarbeitung zu verringern, wobei durch das Motto „Hilfe für sich selbst und andere“ Wert auf die Gleichberechtigung aller Mitglieder gelegt wird und Informations- und Erfahrungsaustausch sowie gemeinsame Aktivitäten im Vordergrund stehen [31]. Die Geschäftsstelle des Deutschen Psoriasis Bundes e.V. in Hamburg informiert interessierte Betroffene und Angehörige über regional organisierte Selbsthilfegruppen.

\section{Fazit für die Praxis}

Für in der Klinik tätige und niedergelassene Dermatologinnen und Dermatologen ergeben sich die folgenden wesentlichen Konsequenzen aus den beschriebenen empirischen Studien für die Arbeit mit Psoriasis-Patientinnen und -Patienten: psychosoziale Belastungen durch die Psoriasis können auch bei eher kleiner Fläche der erkrankten Hautstellen - speziell bei exponierter Lokalisation - auftreten. Deshalb sollten die behandelnden Dermatologen die psychosozialen Beeinträchtigungen durch Schwierigkeiten bei der Beeinflussung der Kleidungswahl, beim Frisör, im Schwimmbad, beim Sport und im Sexualleben auch schon bei relativ geringer Ausdehnung der Hauterkrankung wiederholt thematisieren, da selbst das Betroffensein auch der „unsichtbaren Regionen“ für die mit der Psoriasis einhergehenden psychosozialen Einschränkungen eine erhebliche Bedeutung erhält. Außerdem ist die bei einer Komorbidität mit einer Depression und mit einer Fibromyalgie erheblich erhöhte psychische Belastung von Psoriasis-Patienten zu beachten. Patienten sollten daher auf die Möglichkeit einer Patientenschulung mit dem Ziel der Erweiterung ihres Handlungswissens in Bezug auf die Krankheit aufmerksam gemacht und zur Teilnahme an einer Selbsthilfeorganisation ermutigt werden, damit sie aktiver den psychosozialen Konsequenzen der Psoriasis entgegen wirken können.

\section{Literatur}

${ }^{1}$ Schmid-Ott G. Das sichtbare und das unsichtbare Stigma - Stigmatisierung bei der Psoriasis vulgaris und bei psychosomatischen Erkrankungen. Medizinische Hochschule Hannover: Habilitationsarbeit, 2000

2 Stangier U. Feldstudien zur belastungsbedingten Reaktivität bei Hauterkrankungen. Verhaltensmodifikation und Verhaltensmedizin 1995; 16: $353-371$

${ }^{3}$ Schmid-Ott G, Jacobs R, Jäger B, Klages S, Wolf J, Werfel T, Kapp A, Schürmeyer T, Lamprecht F, Schmidt RE, Schedlowski M. Stress-induced endocrine and immunological changes in psoriasis patients and healthy controls: A preliminary study. Psychother Psychosom 1998; 67: $37-42$

${ }^{4}$ Schmid-Ott G, Jäger B, Adamek C, Koch H, Lamprecht F, Kapp A, Werfel T. Levels of circulating $\mathrm{CD}^{+} \mathrm{T}$ lymphocytes, natural killer cells, and eosinophils increase upon acute psychosocial stress in patients with atopic dermatitis. J Allergy Clin Immunol 2001; 107: 171 - 177

${ }^{5}$ Kurzen H. Die Haut als Spiegel der Seele. Fakt oder Fiktion? Akt Dermatol 2005; 10: 431 - 439

${ }^{6}$ Niemeier V, Klein H, Gieler U, Schill W-B, Kupfer J. Stress und Psoriasis - eine psychoneuroimmunologische Studie. Psychother Psychosom Med Psychol 2005; 55: 20-28
${ }^{7}$ Lebwohl M. Psoriasis. The Lancet 2003; 361: 1197-204

${ }^{8}$ Schmid-Ott G. Future Trends in Psychodermatological Psoriasis Research: Somatopsychic or Psychosomatic Focus? Dermatol Psychosom 2003; 4: 129-130

${ }^{9}$ Krueger G, Koo J, Lebwohl M, Meuter A, Stern RS, Rolstad T. The impact of psoriasis on quality of life: Results of a 1998 National Psoriasis foundation patient-membership survey. Arch Dermatol 2001; 137: $280-284$

${ }^{10}$ Schmid-Ott G, Burchard R, Niederauer HH, Lamprecht F, Künsebeck HW. Stigmatisierungsgefühl und Lebensqualität bei Patienten mit Psoriasis und Neurodermitis. Hautarzt 2003; 54: $852-857$

${ }^{11}$ Updike J. Selbst-Bewußstsein. Reinbek: Rowohlt, 1990: 68

${ }^{12}$ Finlay AY, Khan GK, Luscombe DK, Salek MS. Validation of sickness impact profile and psoriasis disability index in psoriasis. Br J Dermatol 1990; 123: 751 - 756

${ }^{13}$ Schmid-Ott G, Malewski P, Kreiselmaier I, Mrowietz U. Psychosoziale Folgen der Psoriasis. Hautarzt 2005; 56: 466 - 472

${ }^{14}$ Schmid-Ott G, Jäger B, Künsebeck HW, Ott R, Wedderer K, Lamprecht F. Entwicklung des „Fragebogens zum Erleben von Hautbeschwerden“ (FEH): Faktorenanalyse und Untersuchung von Prädiktoren für das Krankheitserleben von Psoriasis-Patienten. ZKPP (Zeitschrift für Klinische Psychologie, Psychiatrie und Psychotherapie) 1998; 46: 330 -343

${ }^{15}$ Epstein SA, Kay G, Clauw D, Heaton R, Klein D, Krupp L, Kuck J, Leslie V, Masur D, Wagner M, Waid R, Zisook S. Psychiatric disorders in patients with fibromyalgia. A multicenter investigation. Psychosomatics 1999; 40: 7-63

${ }^{16}$ Fortune DG, Richards HL, Main CJ, Griffiths CE. Patients' strategies for coping with psoriasis. Clin Exp Dermatol 2002; 27: 177-184

${ }^{17}$ Braun-Falco O, Plewig G, Wolff HH. Dermatologie und Venerologie. 4. Aufl. Berlin: Springer, 1996

18 Stern RS, Nijsten T, Feldman SR, Margolis DJ, Rolstad T. Psoriasis is common and carries a substantial burden even when not extensive and is associated with widespread treatment dissatisfaction. J Investig Dermatol Symp Proc 2004; 9: 136 - 139

${ }^{19}$ Ginsburg IH, Link BG. Feelings of stigmatization in patients with psoriasis. J Am Acad Dermatol 1989; 20: $53-63$

${ }^{20}$ Perrott SB, Murray AH, Lowe J, Mathieson CM. The psychosocial impact of psoriasis: physical severity, quality of life, and stigmatization. Physiol Behav 2000; 70: 567-571

${ }^{21}$ Richards HL, Fortune DG, Griffiths CE, Main CJ. The contribution of perceptions of stigmatisation to disability in patients with psoriasis. J Psychosom Res 2001; 50: $11-15$

22 Vardy D, Besser A, Amir M, Gesthalter B, Biton A, Buskila D. Experiences of stigmatization play a role in mediating the impact of disease severity on quality of life in psoriasis patients. Br J Dermatol 2002; 147: $736-742$

${ }^{23}$ Schmid-Ott G. Dermatologie. In: Adler R, Herrmann JM, Köhle K, Schonecke OW, Uexküll Th von, Wesiack W (Hrsg.). Psychosomatische Medizin / Th. v. Uexküll. 6. Auflage. München: Urban \& Fischer, 2003: $1159-1168$

${ }^{24}$ Richards HL, Fortune DG, Main CJ, Griffiths CEM. Stigmatization and psoriasis. Br J Dermatol 2003; 149: 209-211

25 Niemeier V, Nippesen M, Kupfer J et al. Psychological factors associated with hand dermatoses: which subgroup needs additional psychological care? Br J Dermatol 2002; 146: 1031 - 1037

${ }^{26}$ Schmid-Ott G, Malewski P, Künsebeck HW et al. Krankheitsbezogener Streß bei der Psoriasis vulgaris. Validierung des „Psoriasis-Alltagsstreß-Inventars“ (PAI). Hautarzt 2000; 51: 496-501

${ }^{27}$ Rapp SR, Feldman SR, Exum ML et al. Psoriasis causes as much disability as other major medical diseases. J Am Acad Dermatol 1999; 41: $401-407$

${ }^{28}$ Schmid-Ott G. Stigmatisierung von Hauterkrankten. In: Petermann F, Warschburger P (Hrsg). Neurodermitis. Göttingen: Hogrefe, 1999: $157-174$

${ }^{29}$ Schmid-Ott G, Jodehl S. Rehabilitation und Schulung für Psoriasispatienten. Akt Dermatol 2003; 29: 524-528

${ }^{30}$ Schon MP, Boehncke WH. Psoriasis. N Engl J Med 2005; 352: 1899 1912

${ }^{31}$ Mrowietz U, Schmid-Ott G. Schuppenflechte. Was Sie schon immer über Psoriasis wissen wollten - ein Ratgeber. 2. Auflage. Basel: Karger, 2005 